\title{
Strategisches Innovationsmarketing
}

$\mathrm{D}$ er technologische Wandel ist der größte Wachstumsmotor für eine entwickelte Volkswirtschaft. Innovationen zum Aufbau, Ausbau oder zur Sicherung der Wettbewerbsposition sind für wettbewerbsfähige Unternehmen unerlässlich geworden. Doch um neue attraktive Märkte zu kreieren und auszubauen, müssen Unternehmen die Kompetenz besitzen, Produkte dreimal zu ,erfinden“:

1. als neuartige Bündel von Hardware und Services,

2. als neuartige Konstruktionen von Präferenzen in den „Köpfen“ der Kunden und

3. als Instrument zur Bekämpfung des Wettbewerbs.

Um neuartige Bündel von Hardware und Services erfinden zu können, reicht die Entwicklung von Produkten durch die interne F\&E-Abteilung allein längst nicht mehr aus. Stattdessen gilt es, alles vorhandene Wissen zu nutzen und während des gesamten Innovationsprozesses systematisch einzubinden. Der Ansatz wechselt bei Procter \& Gamble von ,the lab is our world“ $\mathrm{zu}$,the world is our lab“. Deshalb diskutieren wir in der Rubrik „Kollaboration“ Chancen, Herausforderungen und Methoden von Open-InnovationProzessen. Erfolgreich können solche Prozesse sein, wenn eine Balance gehalten wird zwischen internen und externen Entwicklungen durch Kooperationen, Kundenintegration, Netzwerkaktivitäten und die Multiplikation eigener Technologien und Kompetenzen in neue Märkte.

Doch selbst wenn eine Innovation erfolgreich entwickelt wurde - Nachahmer lassen meist nicht lange auf sich warten. Um das Produkt als Schutz gegen den Wettbewerb zu „erfinden“, bedarf es nicht nur der rechtzeitigen Anmeldung von Patenten oder Markenrechten. Der globale Schutz vor Fälschungen erfordert weitere Maßnahmen. Deshalb greift ein Beitrag in der zweiten Rubrik „Wettbewerb und Kunde“ das Phänomen des sogenannten „Counterfeiting“ auf und erläutert Strategien zum Schutz vor chinesischen Fälschungen. Darüber hinaus wird vertieft, wie der Kunde selbst zum Innovationsmotor werden kann. Indem Unternehmen die Bedürfnisse ihrer aktuellen und potenziellen Kunden frühzeitig erkennen, kann es ihnen gelingen, ein Nutzenbündel zu kreieren, das Kunden gegenüber Wettbewerbsprodukten und -dienstleistungen bevorzugen.

Um die Präferenz in den Köpfen der Kunden zu schaffen, bedarf es aber nicht nur eines einzigartigen Nutzenbündels, sondern auch der professionellen Innovationskommunikation. Deshalb greift die dritte Rubrik „Kommunikation“ die neuen Rollen der Marketingfachleute im Zeitalter der Open Innovation auf. Zunehmend schlüpfen Marketingverantwortliche in die Rolle der Meinungsbeobachter und Mediatoren zwischen F\&E-Abteilungen und externen Bezugsgruppen; sie müssen komplexe Sachverhalte kreativ und verständlich an interne und externe Stakeholder vermitteln. Das Beispiel Apple iPhone zeigt eindrücklich, wie es dem Unternehmen gelang, nicht nur ästhetische und emotionalisierende Faktoren in den Vordergrund zu stellen, sondern den Nutzen des iPhones verständlich zu kommunizieren und damit das Produkt erfolgreich am Markt zu positionieren.

Die Zusammenstellung dieser Beiträge zum strategischen Innovationsmarketing verspricht einen breit angelegten Blick auf das Thema und vielfältige Impulse für Marketingverantwortliche. Wir wünschen Ihnen eine interessante Lektüre.

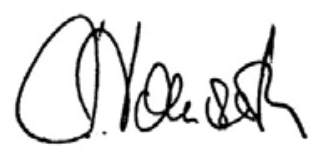

Prof. Dr. Torsten Tomczak

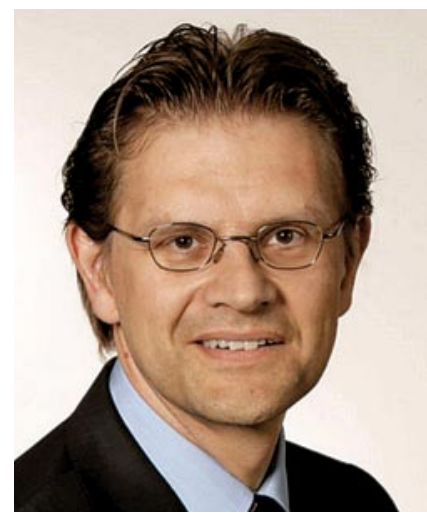

Torsten Tomczak Prof. Dr. Torsten Tomczak, Ordinarius für Betriebswirtschaftslehre mit besonderer Berücksichtigung des Marketing und Direktor der Forschungsstelle für Customer Insight an der Universität St. Gallen E-Mail: torsten.tomczak@unisg.ch

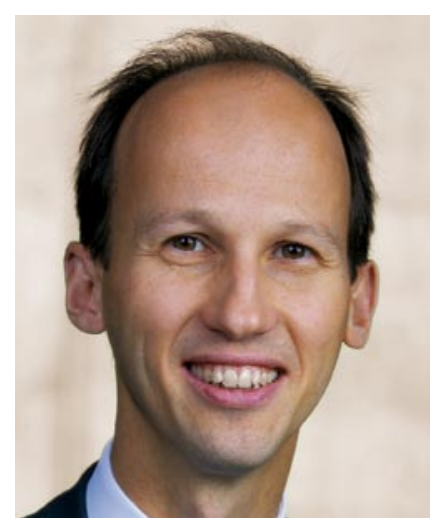

Oliver Gassmann

Prof. Dr. Oliver Gassmann, Ordinarius für Technologie- und Innovationsmanagement an der Universität St. Gallen und Vorsitzender der Direktion des Instituts für Technologiemanagement

E-Mail: oliver.gassmann@unisg.ch 